\title{
Quantum effective potential from discarded degrees of freedom
}

\author{
Luke M. Butcher* \\ Institute for Astronomy, University of Edinburgh, \\ Royal Observatory, Edinburgh EH9 3HJ, United Kingdom
}

(Dated: July 10, 2018)

\begin{abstract}
I obtain the quantum correction $\Delta V_{\text {eff }}=\left(\hbar^{2} / 8 m\right)\left[\left(1-4 \xi \frac{d+1}{d}\right)\left(\mathcal{S}^{\prime}\right)^{2}+2(1-4 \xi) \mathcal{S}^{\prime \prime}\right]$ that appears in the effective potential whenever a compact $d$-dimensional subspace (of volume $\propto \exp [\mathcal{S}(x)]$ ) is discarded from the configuration space of a nonrelativistic particle of mass $m$ and curvature coupling parameter $\xi$. This correction gives rise to a force $-\left\langle\Delta V_{\text {eff }}^{\prime}\right\rangle$ that pushes the expectation value $\langle x\rangle$ off its classical trajectory. Because $\Delta V_{\text {eff }}$ does not depend on the details of the discarded subspace, these results constitute a generic model of the quantum effect of discarded variables with maximum entropy/information capacity $\mathcal{S}(x)$.
\end{abstract}

\section{INTRODUCTION}

It is often possible and desirable to ignore specific degrees of freedom of a system, and focus on those that remain. For example, consider a nonrelativistic particle in a curved two-dimensional space

$$
\mathrm{d} s^{2}=\mathrm{d} x^{2}+[b(x)]^{2} \mathrm{~d} \phi^{2}, \quad(x, \phi) \in \mathbb{R} \times[0,2 \pi),
$$

as illustrated in figure 1. If the particle also encounters a potential $V_{0}(x)$ then its action is

$$
\mathcal{I}[x(t), \phi(t)]=\int \mathrm{d} t\left[\frac{m}{2}\left(\dot{x}^{2}+b^{2} \dot{\phi}^{2}\right)-V_{0}\right],
$$

giving rise to the following equations of motion:

$$
\begin{aligned}
m \ddot{x} & =m b^{\prime} b \dot{\phi}^{2}-V_{0}^{\prime}, \\
m b^{2} \dot{\phi} & =p_{\phi}=\text { const. }
\end{aligned}
$$

Now suppose we only wish to describe the behaviour of the $x$ coordinate of this particle - perhaps $\phi$ is unobservable in practice, or happens to be irrelevant to whatever applications we have in mind. At the classical level, we can separate the $x$-motion from the $\phi$-motion as follows. Let us write the action (2) as

$$
\begin{aligned}
\mathcal{I}[x(t), \phi(t)]= & \int \mathrm{d} t\left[\frac{m}{2} \dot{x}^{2}+\frac{1}{2 m b^{2}}\left(m b^{2} \dot{\phi}-p_{\phi}\right)^{2}\right. \\
& \left.+\dot{\phi} p_{\phi}-\frac{p_{\phi}^{2}}{2 m b^{2}}-V_{0}\right],
\end{aligned}
$$

and note that

$$
\begin{aligned}
& \frac{\delta}{\delta x(t)} \int \mathrm{d} t\left[\frac{1}{2 m b^{2}}\left(m b^{2} \dot{\phi}-p_{\phi}\right)^{2}+\dot{\phi} p_{\phi}\right] \\
& =-\frac{b^{\prime}}{m b^{3}}\left(m b^{2} \dot{\phi}-p_{\phi}\right)^{2}+\frac{2 b^{\prime} \dot{\phi}}{b}\left(m b^{2} \dot{\phi}-p_{\phi}\right),
\end{aligned}
$$

which vanishes on the $\delta / \delta \phi$ equation of motion (4). If we only want to determine $x(t)$, we can therefore discard the

\footnotetext{
*lmb@roe.ac.uk
}

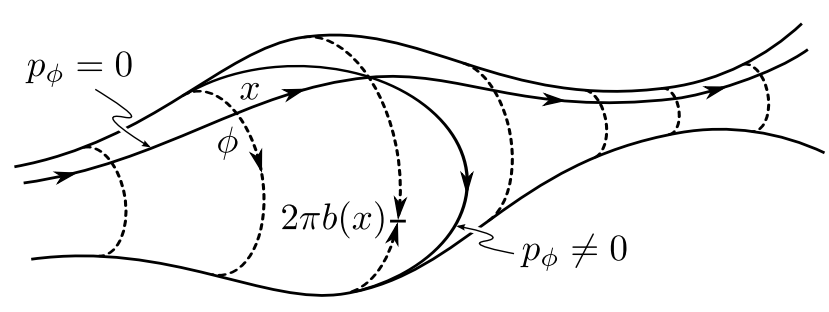

FIG. 1. As classical particles move over the curved space (1) their $x$ coordinate can be predicted without reference to $\phi$, using the reduced action (7). However, quantum particles encounter an addition potential $\Delta V_{\text {eff }}$ due to variations in the physical size $2 \pi b$ of the discarded subspace $\phi \in[0,2 \pi)$.

second and third terms in (5) and work with the reduced action:

$$
\mathcal{I}[x(t)] \equiv \int \mathrm{d} t\left[\frac{m}{2} \dot{x}^{2}-V_{\mathrm{cl}}\right],
$$

where the (classical) effective potential is

$$
V_{\mathrm{cl}}=V_{0}+\frac{p_{\phi}^{2}}{2 m b^{2}} .
$$

The reduced action (7) generates the correct equations of motion for $x$, consistent with substituting (4) into (3), and allows us to treat the particle as though it were living in a reduced configuration space

$$
\mathrm{d} s^{2}=\mathrm{d} x^{2}, \quad x \in \mathbb{R} .
$$

We no longer need to refer to $\phi$, and can think of $p_{\phi}$ as a parameter of the system. For a concrete application of this formalism, recall Newtonian orbital mechanics: with $b(x)=x$, the metric (1) describes a flat plane with radial coordinate $x$, and $V_{\mathrm{cl}}=V_{0}+p_{\phi}^{2} / 2 m x^{2}$ is the standard centrifugal potential.

It is important to realise, however, that once quantum effects are considered, the above procedure is no longer valid. If we naïvely quantize the reduced system (7) we will not arrive at the correct result: that obtained by quantizing the original system (2) and then reducing its configuration space. As we will see, the correct result 
differs from the naïve one by a quantum correction to the effective potential $\Delta V_{\text {eff }}$, dependent on the physical size $\mathrm{Vol}_{\phi}=2 \pi b(x)$ of the discarded subspace $\mathcal{M}_{\phi} \cong[0,2 \pi)$.

\section{DISCARDING A SINGLE VARIABLE}

Let us work in the Schrödinger picture, and first confirm the existence of $\Delta V_{\text {eff }}$ for the simple system above. As usual, we describe the quantum particle with a wavefunction, a scalar field $\Psi(x, \phi, t)$ that defines coordinateinvariant probabilities via integrals of the form

$$
P=\int \mathrm{d} x \mathrm{~d} \phi \sqrt{g}|\Psi|^{2},
$$

where $\sqrt{g} \equiv \sqrt{\operatorname{det}\left(g_{i j}\right)}$ is the covariant measure endowed by the metric $g_{i j}$. [(1) $\Rightarrow \sqrt{g}=b$.] In curved space, $\Psi$ obeys the covariant Schrödinger equation ${ }^{1}$ :

$$
i \hbar \partial_{t} \Psi=\left[\frac{\hbar^{2}}{2 m}\left(-\nabla^{2}+\xi R\right)+V_{0}\right] \Psi
$$

where the Laplacian

$$
\nabla^{2}=\frac{1}{\sqrt{g}} \partial_{i} \sqrt{g} g^{i j} \partial_{j}
$$

and the Ricci scalar $R \equiv R_{i j} g^{i j} \equiv R^{k}{ }_{i k j} g^{i j}$ are constructed from the metric $g_{i j} .{ }^{2}\left[(1) \Rightarrow R=-2 b^{\prime \prime} / b\right.$.] The form of (11) is fixed by coordinate invariance, unitarity, locality, dimensional considerations, and the limits $R \rightarrow 0, V_{0} \rightarrow 0$; however, the curvature coupling parameter $\xi \in \mathbb{R}$ is an arbitrary dimensionless constant, representing a quantization ambiguity of the system [1$3]$. One can choose to invoke 'minimal coupling' $\xi=0$, or motivate a conformal coupling according to some other principle or consideration $[9,10]$. For the sake of generality, we leave $\xi$ unspecified.

Having quantized the original system, we proceed to discard the $\phi$ subspace. In order to make $p_{\phi}$ a parameter of the system, we must first insist that the particle be in an eigenstate of the angular momentum operator:

$$
\hat{p}_{\phi} \Psi \equiv-i \hbar \partial_{\phi} \Psi=p_{\phi} \Psi .
$$

This requirement serves as the analogue of equation (4) and ensures that the particle's $\phi$ behaviour is sufficiently

\footnotetext{
1 The first systematic treatment of quantum mechanics in curved space is due to DeWitt [1] whose paper includes a canonical derivation of the covariant Schrödinger equation. For another perspective on the origin and ambiguity of the curvature term, see [2]. A more modern approach can be found in [3].

2 The curved tube (1) is the entire configuration space of the system, so the covariant Schrödinger equation (11) can refer only to the intrinsic geometry of this manifold. Contrast this with a particle that actually exists in $\mathbb{R}^{3}$, but is constrained to a twodimensional surface $\Sigma \subset \mathbb{R}^{3}$ by a steep potential well: here, the extrinsic curvature of $\Sigma$ will also play a role [4-8].
}

simple that the $x$ dynamics can be described in isolation. The states of interest are then

$$
\Psi=\frac{e^{i k \phi}}{\sqrt{2 \pi b(x)}} \Psi_{x}(x, t), \quad p_{\phi} / \hbar=k \in \mathbb{Z},
$$

where the normalisation of $\Psi_{x}$ ensures that probabilities (10) become integrals of the form

$$
P=\int \mathrm{d} x\left|\Psi_{x}\right|^{2}
$$

without any reference to the $\phi$ subspace. Hence we can think of $\Psi_{x}$ as the wavefunction of the particle on the reduced configuration space (9).

To obtain the evolution equation for $\Psi_{x}$, we simply insert (14) into (11). We arrive at a reduced Schrödinger equation

$$
i \hbar \partial_{t} \Psi_{x}=\left[-\frac{\hbar^{2}}{2 m} \partial_{x}^{2}+V_{\mathrm{qu}}\right] \Psi_{x}
$$

where the quantum effective potential

$$
V_{\mathrm{qu}} \equiv V_{\mathrm{cl}}+\Delta V_{\mathrm{eff}}
$$

has an additional contribution

$$
\Delta V_{\text {eff }}=\frac{\hbar^{2}}{2 m}\left[-\frac{1}{4}\left(\frac{b^{\prime}}{b}\right)^{2}+\frac{1-4 \xi}{2}\left(\frac{b^{\prime \prime}}{b}\right)\right]
$$

as promised. There are a few things to note about this quantum correction. First, this effect is not purely a consequence of spatial curvature: even for the flat case $b=x$ we find $\Delta V_{\text {eff }} \neq 0$. Second, $\Delta V_{\text {eff }}$ cannot be made to vanish identically by some choice of $\xi$. Third, $\Delta V_{\text {eff }}$ does not depend on $p_{\phi}$, so all states (14) experience the same correction.

To illustrate an important physical implication of $\Delta V_{\text {eff }}$, we now consider an arbitrary state:

$$
\Psi=\sum_{k=-\infty}^{\infty} \frac{e^{i k \phi}}{\sqrt{2 \pi b(x)}} \Psi_{x}^{k}(x, t)
$$

where $\int \mathrm{d} x \sum_{k}\left|\Psi_{x}^{k}\right|^{2}=1$ ensures proper normalisation. The expectation value of a function $f\left(x, p_{\phi}\right)$ is then

$$
\begin{aligned}
\left\langle f\left(x, p_{\phi}\right)\right\rangle & \equiv \int \mathrm{d} x \mathrm{~d} \phi \sqrt{g} \Psi^{*} f\left(x,-i \hbar \partial_{\phi}\right) \Psi \\
& =\int \mathrm{d} x \sum_{k=-\infty}^{\infty}\left|\Psi_{x}^{k}\right|^{2} f(x, \hbar k) .
\end{aligned}
$$

As each $\Psi_{x}^{k}$ obeys the reduced Schrödinger equation (16) with $p_{\phi}=\hbar k$, it follows that $\langle x\rangle$ evolves according to

$$
m \partial_{t}^{2}\langle x\rangle=-\left\langle V_{\mathrm{cl}}^{\prime}+\Delta V_{\mathrm{eff}}^{\prime}\right\rangle .
$$


We see that the classical equation of motion $m \ddot{x}=-V_{\mathrm{cl}}^{\prime}$ no longer holds true on average. Indeed, the average deviation from the classical equation is given by

$$
\begin{aligned}
m \partial_{t}^{2}\langle x\rangle+\left\langle V_{\mathrm{cl}}^{\prime}\right\rangle & =-\left\langle\Delta V_{\text {eff }}^{\prime}\right\rangle \\
& =-\int \mathrm{d} x \Delta V_{\text {eff }}^{\prime} \sum_{k=-\infty}^{\infty}\left|\Psi_{x}^{k}\right|^{2},
\end{aligned}
$$

which only depends on $\Delta V_{\text {eff }}^{\prime}$ and the probability density over the reduced configuration space:

$$
\rho_{x}(x, t) \equiv \int \mathrm{d} \phi \sqrt{g}|\Psi|^{2}=\sum_{k=-\infty}^{\infty}\left|\Psi_{x}^{k}\right|^{2}
$$

Hence $\Delta V_{\text {eff }}$ directly influences the average motion of the particle, independent of the internal details of the quantum state.

Had we naïvely quantized the reduced system (7) we would not have included the quantum contribution (18) to our effective potential. Moreover, nothing about the reduced action (7) nor the configuration space (9) would have hinted at the error we were making - we would encounter no striking technical difficulties or operator ambiguities. As such, this result serves as a general warning to those attempting to quantize any system in which degrees of freedom have already been discarded: if the physical size of the discarded configuration space varies as a function of the remaining variables, then one expects to miss an effective potential similar to (18). For instance, the mini-superspace approach to quantum cosmology [11] will need to quantify the volume of configuration space neglected in assuming a highly symmetric universe.

\section{DISCARDING A GENERIC SUBSPACE}

Thus far, we have obtained the quantum correction (18) that arises from the removal of a one-dimensional subspace. To generalise this result, let us now consider a nonrelativistic quantum particle in $D=d+1$ spatial dimensions:

$$
\mathrm{d} s^{2}=\mathrm{d} x^{2}+[b(x)]^{2} \tilde{g}_{I J}(\phi) \mathrm{d} \phi^{I} \mathrm{~d} \phi^{J},
$$

where $\tilde{g}_{I J}$ is the metric of a compact $d$-dimensional manifold $\mathcal{M}_{\phi}$ with coordinates $\phi \equiv\left(\phi^{1}, \ldots, \phi^{d}\right)$. As before, we seek a description of the dynamics in which we can ignore $\phi$ and treat the particle as though it were living in the reduced configuration space (9) with an effective potential that depends on a single parameter. In fact (24) is the most general metric that allows for this type of reduction: see the appendix for a proof.

At the classical level, the analysis follows steps (2)-(8) with minor modifications. In place of $p_{\phi}$, we assemble

$$
E_{\phi} \equiv \frac{1}{2 m} \tilde{g}^{I J} p_{\phi^{I}} p_{\phi^{J}}=\frac{m b^{4}}{2} \tilde{g}_{I J} \dot{\phi}^{I} \dot{\phi}^{J}
$$

which is conserved by virtue of the $\delta / \delta \phi^{I}$ equations of motion. Writing $|\dot{\phi}| \equiv\left(\tilde{g}_{I J} \dot{\phi}^{I} \dot{\phi}^{J}\right)^{1 / 2}$, we therefore have

$$
m b^{2}|\dot{\phi}|=\sqrt{2 m E_{\phi}}=\text { const }
$$

as a substitute for equation (4). Hence steps (5) and (6) now follow with replacements $\dot{\phi} \rightarrow|\dot{\phi}|, p_{\phi} \rightarrow \sqrt{2 m E_{\phi}}$. This generates the reduced action (7) with

$$
V_{\mathrm{cl}}=V_{0}+E_{\phi} / b^{2}
$$

as the classical effective potential.

On the quantum side, we begin by observing that the Laplacian and Ricci scalar can be decomposed as follows:

$$
\begin{aligned}
\nabla^{2} & =b^{-d} \partial_{x} b^{d} \partial_{x}+b^{-2} \tilde{\nabla}^{2}, \\
R & =d(1-d)\left(b^{\prime} / b\right)^{2}-2 d\left(b^{\prime \prime} / b\right)+\tilde{R} / b^{2},
\end{aligned}
$$

where $\tilde{\nabla}^{2}$ and $\tilde{R}$ are constructed from the metric $\tilde{g}_{I J}$. The states (14) generalise to

$$
\Psi=\frac{\Phi(\phi)}{[b(x)]^{d / 2}} \Psi_{x}(x, t),
$$

where $\Phi$ is an 'energy' eigenfunction on $\mathcal{M}_{\phi}$,

$$
\frac{\hbar^{2}}{2 m}\left(-\tilde{\nabla}^{2}+\xi \tilde{R}\right) \Phi=E_{\phi} \Phi,
$$

with unit norm:

$$
\int \mathrm{d}^{d} \phi \sqrt{\tilde{g}}|\Phi|^{2}=1 .
$$

It is natural to identify $E_{\phi}$, defined in (25), with the eigenvalue of (31) because the covariant Schrödinger equation (11) follows the same quantisation rule $g^{i j} p_{i} p_{j} \rightarrow \hbar^{2}\left[-\nabla^{2}+\xi R\right]$. As before, we have normalised $\Psi_{x}$ such that probabilities have the standard form (15).

Inserting (28)-(31) into the Schrödinger equation (11) we obtain the reduced Schrödinger equation (16) once again. The quantum effective potential (17) now differs from its classical counterpart (27) by

$$
\begin{aligned}
\Delta V_{\mathrm{eff}}= & \frac{\hbar^{2} d}{2 m}\left[\left(\frac{d-2}{4}+\xi(1-d)\right)\left(\frac{b^{\prime}}{b}\right)^{2}\right. \\
& \left.+\frac{1-4 \xi}{2}\left(\frac{b^{\prime \prime}}{b}\right)\right] .
\end{aligned}
$$

We see that $\Delta V_{\text {eff }}$ is independent of $\tilde{g}_{I J}$ and $E_{\phi}$, and does not vanish identically for any $(\xi, d) \in \mathbb{R} \times \mathbb{N}$.

It is easy to check that $\Delta V_{\text {eff }}$ appears in the average equation of motion (21) just as before. The only change to this calculation is that the arbitrary state (19) is now

$$
\Psi=\sum_{k} \frac{\Phi_{k}(\phi)}{[b(x)]^{d / 2}} \Psi_{x}^{k}(x, t)
$$


where the $\left\{\Phi_{k}\right\}$ are eigenfunctions (31) with eigenvalues $\left\{E_{\phi}^{k}\right\}$, forming an orthonormal basis over $\mathcal{M}_{\phi}$ :

$$
\int \mathrm{d}^{d} \phi \sqrt{\tilde{g}} \Phi_{k}^{*} \Phi_{k^{\prime}}=\delta_{k k^{\prime}}
$$

Consequently, we compute expectation values with

$$
\begin{aligned}
\left\langle f\left(x, E_{\phi}\right)\right\rangle & \equiv \int \mathrm{d} x \mathrm{~d}^{d} \phi \sqrt{g} \Psi^{*} f\left(x, \frac{\hbar^{2}}{2 m}\left[-\tilde{\nabla}^{2}+\xi \tilde{R}\right]\right) \Psi \\
& =\int \mathrm{d} x \sum_{k}\left|\Psi_{x}^{k}\right|^{2} f\left(x, E_{\phi}^{k}\right)
\end{aligned}
$$

instead of equation (20).

\section{DISCARDED INFORMATION CAPACITY}

The power of equation (33) is revealed by expressing this result in terms of the information we discard by ignoring the degrees of freedom in $\phi$. To quantify this information, we first need to regularise the infinitedimensional Hilbert space of the particle. Let us imagine dividing the curved space (24) into a lattice of small cells with spacing $\ell \ll \min \left\{b,\left(b / b^{\prime}\right), \sqrt{b / b^{\prime \prime}}\right\}$. Then, at a given value of $x$, the particle can be in any of

$$
\Omega(x)=\frac{\operatorname{Vol}_{\phi}(x)}{\ell^{d}} \propto \frac{[b(x)]^{d}}{\ell^{d}}
$$

locations on the $\phi$ sub-lattice, and the maximum entropy (or information) that can be stored in the $\phi$ subspace is given by Boltzmann's formula:

$$
\mathcal{S}=\ln \Omega
$$

With this in mind, equation (33) can be written as

$$
\Delta V_{\text {eff }}=\frac{\hbar^{2}}{8 m}\left[\left(1-4 \xi \frac{d+1}{d}\right)\left(\mathcal{S}^{\prime}\right)^{2}+2(1-4 \xi) \mathcal{S}^{\prime \prime}\right] \text {. }
$$

Crucially, this formula is completely independent of the arbitrary length $\ell$. If we wish, we can now take $\ell \rightarrow 0$ and safely return to the continuum limit. Thus we have obtained a robust relation between the quantum correction and the maximum entropy/information capacity $\mathcal{S}$ of the discarded subspace.

\section{SEMICLASSICAL ACTION}

Because $\Delta V_{\text {eff }}$ does not depend on the details of the discarded space $\mathcal{M}_{\phi}$, equation (39) can be used to model discarded variables in general. To illustrate this idea, suppose we are interested in predicting the behaviour of an observable $x$ in a system which is not well-understood, but is known to have the following two properties. First, the classical motion $x(t)$ can be derived from an action
(7) without reference to other dynamical variables. Second, for a given value of $x$, the system can store exactly $\mathcal{S}(x)$ nats of information. Now, even if we know nothing about the 'discardable' degrees of freedom that hold the information, we can still model their effect on the quantum behaviour of $x$. The method is simple: treat the full configuration space as (24) and leave the internal metric $\tilde{g}_{I J}$ unspecified. We conclude that the informationholding variables introduce a quantum correction (39) to the effective potential, regardless of the details of their configuration space. Furthermore, we can account for this effect semiclassically by replacing the classical action (7) with

$$
\mathcal{J}[x(t)]=\int \mathrm{d} t\left[\frac{m}{2} \dot{x}^{2}-\left(V_{\mathrm{cl}}+\Delta V_{\text {eff }}\right)\right],
$$

which generates equations that accurately capture the motion $(21)$ of the expectation value $\langle x\rangle$. Provided $\mathcal{S}(x)$ is known, the semiclassical action (40) only introduces two parameters $(\xi, d)$ that would need to be determined experimentally. Thus equations (39) and (40) constitute a powerful semiclassical model of the quantum effect of discarded variables.

The semiclassical action (40) also lets us express the propagator for $\Psi_{x}$ as a path integral over the reduced configuration space:

$$
K\left(x_{f}, x_{0} ; T\right)=\int_{x(0)=x_{0}}^{x(T)=x_{f}} \mathcal{D} x(t) e^{i \mathcal{J}[x(t)] / \hbar} .
$$

This relation is evident from the reduced Schrödinger equation (16) and the standard path integral construction $[12,13]$. Furthermore, it must also be possible to derive the reduced propagator (41) from the path integral over the entire configuration space $\int \mathcal{D} x(t) \mathcal{D}^{d} \phi(t) \ldots$, by 'integrating out' the paths in $\phi .^{3}$ In a future publication, I will demonstrate this process explicitly, and hence provide a derivation of $\Delta V_{\text {eff }}$ and $\mathcal{J}[x(t)]$ that does not require the Schrödinger equation.

\section{CONCLUSIONS}

When predicting the classical motion of an observable $x$, other degrees of freedom can often be ignored and subsumed into an effective potential. However, if these discarded variables have a configuration space which varies in size as a function of $x$, so that the discarded information capacity is $\mathcal{S}(x)$, then quantum effects generate an additional term in the effective potential (39). This quantum correction directly influences the observable behaviour, forcing the expectation value $\langle x\rangle$ away from its

\footnotetext{
3 There is some ambiguity in the definition of this path integral, each resolution of which fixes the value of $\xi$ in the effective potential (39). Storchak has performed a similar treatment of the phase space path integral with $\xi=0$ [14].
} 
classical trajectory (21). The semiclassical action (40) accounts for this phenomenon within the equation of motion, and also generates the path integral propagator (41) over the reduced configuration space (9). In general, the quantum correction is determined by $\mathcal{S}(x)$, the number of discarded variables $d \in \mathbb{N}$, and the curvature coupling parameter $\xi \in \mathbb{R}$; beyond this, the details of the discarded configuration space are irrelevant. As such, these results constitute a powerful general-purpose model for the quantum effect of discarded variables, applicable to any observable whose classical motion is determined by the standard nonrelativistic action (7).

\section{ACKNOWLEDGEMENTS}

The author is supported by a research fellowship from the Royal Commission for the Exhibition of 1851. He also thanks the anonymous reviewer whose comments motivated the appendix.

\section{Appendix A: Reducible Spaces}

We have seen that the $x$-coordinate of a particle can sometimes be predicted without detailed knowledge of the other degrees of freedom: a single parameter (e.g. $p_{\phi}$ or $\left.E_{\phi}\right)$ contains all the information we need. Here, I prove that the only manifolds that allow this kind of reduction are those with a metric of the form (24).

Let us begin with an arbitrary metric in $D=d+1$ spatial dimension:

$$
\mathrm{d} s^{2}=g_{i j}(q) \mathrm{d} q^{i} \mathrm{~d} q^{j},
$$

where the coordinates $q \equiv\left(q^{1}, \ldots, q^{d+1}\right) \equiv$ $\left(x, \phi^{1}, \ldots, \phi^{d}\right)$ cover an open region $\mathcal{U} \cong\left(x_{-}, x_{+}\right) \times \mathcal{U}_{\phi}$, with $\mathcal{U}_{\phi}$ homeomorphic to an open $d$-ball. A particle of mass $m$ moving in $\mathcal{U}$, under the influence of a potential $V_{0}$, will have a Hamiltonian

$$
H=\frac{1}{2 m} g^{i j}(q) p_{i} p_{j}+V_{0}(q)
$$

where $g^{i j}$ is the inverse of $g_{i j}$, and $\left\{p_{i}\right\}$ are the momenta conjugate to $\left\{q^{i}\right\}$.

We aim to discard the coordinates $\phi \equiv\left(\phi^{1}, \ldots, \phi^{d}\right)$ and momenta $p_{\phi} \equiv\left(p_{\phi^{1}}, \ldots, p_{\phi^{d}}\right)$ and predict the motion of $\left(x, p_{x}\right)$ from a one-dimensional Hamiltonian

$$
H=\frac{p_{x}^{2}}{2 \tilde{m}(\lambda)}+V_{\mathrm{cl}}(x, \lambda)
$$

where the effective mass $\tilde{m}$ and effective potential $V_{\mathrm{cl}}$ can depend on a single real parameter $\lambda=\lambda\left(x, p_{x}, \phi, p_{\phi}\right) \in \mathbb{R}$. (We take $\tilde{m}, V_{\mathrm{cl}}$, and $\lambda$ to be $C^{1}$ functions.) In order that $\lambda$ can be treated as a parameter of the system, it must be conserved along every trajectory, and must not spoil the canonical equations of motion:

$$
\dot{x}=\frac{\partial H}{\partial p_{x}}=\frac{p_{x}}{\tilde{m}}, \quad \dot{p}_{x}=-\frac{\partial H}{\partial x}=-\partial_{x} V_{\mathrm{cl}} .
$$

But note that (A3) will generate (A4) if and only if

$$
\frac{\partial H}{\partial \lambda} \frac{\partial \lambda}{\partial x}=\frac{\partial H}{\partial \lambda} \frac{\partial \lambda}{\partial p_{x}}=0
$$

everywhere. Thus, $H$ can depend on $\lambda$ only where $\lambda$ is independent of $\left(x, p_{x}\right)$. It is therefore safe to assume that $\lambda=\lambda\left(\phi, p_{\phi}\right)$, independent of $\left(x, p_{x}\right)$, without any loss in the generality of $H$. Consequently, $\lambda: T^{*} \mathcal{U}_{\phi} \rightarrow \mathbb{R}$ maps the cotangent bundle $T^{*} \mathcal{U}_{\phi} \cong \mathcal{U}_{\phi} \times \mathbb{R}^{d}$ onto

$$
\Lambda \equiv \lambda\left(T^{*} \mathcal{U}_{\phi}\right) \subseteq \mathbb{R}
$$

which represents the range of possible values for $\lambda\left(\phi, p_{\phi}\right)$.

Our task is to determine which geometries (A1) have Hamiltonians (A2) consistent with the reduced form (A3). In other words, we require

$$
\frac{1}{2 m} g^{i j}(q) p_{i} p_{j}+V_{0}(q)=\frac{p_{x}^{2}}{2 \tilde{m}(\lambda)}+V_{\mathrm{cl}}(x, \lambda),
$$

for all $\left(x, \phi, p_{x}, p_{\phi}\right) \in T^{*} \mathcal{U}$. Recalling $\lambda=\lambda\left(\phi, p_{\phi}\right)$, we can compare powers of $p_{x}$ in (A7) and immediately extract

$$
g^{x x}(x, \phi)=\frac{m}{\tilde{m}\left(\lambda\left(\phi, p_{\phi}\right)\right)}, \quad g^{x \phi}=0 .
$$

But given that $g^{x x}$ does not depend on $p_{\phi}$, we must either have $\lambda=\lambda(\phi)$ or $\tilde{m}=$ const. Even in the former case we still have $\tilde{m}=\tilde{m}(\lambda(\phi))=$ const, because $\lambda=\lambda(\phi)$ cannot be conserved along every trajectory unless it is actually independent of $\phi$. Thus $g^{x x}=m / \tilde{m}=$ const, and by rescaling the $x$-coordinate we can always set $g^{x x}=$ 1. Equation (A7) therefore reduces to

$$
\frac{1}{2 m} g^{I J}(x, \phi) p_{\phi^{I}} p_{\phi^{J}}+V_{0}(x, \phi)=V_{\mathrm{cl}}\left(x, \lambda\left(\phi, p_{\phi}\right)\right) .
$$

It will be useful to analyse this equation at a particular value of $x$, say $x=x_{0} \in\left(x_{-}, x_{+}\right)$, and invert the function $f(\cdot) \equiv V_{\mathrm{cl}}\left(x_{0}, \cdot\right)$ on the right-hand side. To this end, we will prove the following statement:

Claim. For $d \geq 2$, a function $f: \Lambda \rightarrow \mathbb{R}$ that satisfies

$$
f\left(\lambda\left(\phi, p_{\phi}\right)\right)=\frac{1}{2 m} g^{I J}\left(x_{0}, \phi\right) p_{\phi^{I}} p_{\phi^{J}}+V_{0}\left(x_{0}, \phi\right),
$$

will be invertible.

Proof. Begin by differentiating (A10) with respect to $p_{\phi^{I}}$ :

$$
\frac{\mathrm{d} f}{\mathrm{~d} \lambda} \frac{\partial \lambda}{\partial p_{\phi^{I}}}=\frac{1}{m} g^{I J}\left(x_{0}, \phi\right) p_{\phi^{J}} .
$$

Thus $\mathrm{d} f / \mathrm{d} \lambda=0 \Rightarrow p_{\phi}=0$, or equivalently

$$
\frac{\mathrm{d} f}{\mathrm{~d} \lambda} \neq 0 \quad \forall \lambda \in \Lambda_{p_{\phi} \neq 0} \equiv \lambda\left(\left[T^{*} \mathcal{U}_{\phi}\right]_{p_{\phi} \neq 0}\right) .
$$


For $d \geq 2$, the set $\left[T^{*} \mathcal{U}_{\phi}\right]_{p_{\phi} \neq 0}$ is connected, and given that $\lambda$ is continuous, $\Lambda_{p_{\phi} \neq 0}$ will be connected also. Thus, by continuity of $d f / d \lambda$ we must have

$$
\begin{aligned}
& \text { either } \frac{\mathrm{d} f}{\mathrm{~d} \lambda}>0 \quad \forall \lambda \in \Lambda_{p_{\phi} \neq 0}, \\
& \text { or } \frac{\mathrm{d} f}{\mathrm{~d} \lambda}<0 \quad \forall \lambda \in \Lambda_{p_{\phi} \neq 0} \text {. }
\end{aligned}
$$

We will treat the first case only - the second possibility can be dealt with in a similar fashion.

To proceed, we shall construct paths in $\left[T^{*} \mathcal{U}_{\phi}\right]_{p_{\phi} \neq 0}$ that explore all $\lambda\left(\phi, p_{\phi}\right) \in \Lambda$ (except perhaps the upper and lower bounds) and then appeal to monotonicity (A13) to prove that $f$ is invertible. According to the definition (A6) for each $\lambda^{\star} \in \Lambda$ there is at least one $\left(\phi^{\star}, p_{\phi}^{\star}\right) \in T^{*} \mathcal{U}_{\phi}$ such that $\lambda\left(\phi^{\star}, p_{\phi}^{\star}\right)=\lambda^{\star}$. So for every $\lambda^{\star} \in \Lambda$, we can always define a path

$$
\Gamma: \mathbb{R} \rightarrow\left[T^{*} \mathcal{U}_{\phi}\right]_{p_{\phi} \neq 0}, \quad \Gamma(s) \equiv\left(\phi^{\star}, p_{\phi}^{\star}+e^{s} v\right),
$$

where we choose $v=\left(v_{1}, \ldots, v_{d}\right) \neq 0$ such that $v \cdot p_{\phi}^{\star} \geq 0$. Observe that the path (A15) has the following properties:

$$
\begin{aligned}
\lambda(\Gamma(\mathbb{R})) & \subseteq \Lambda_{p_{\phi} \neq 0}, \\
\lim _{s \rightarrow-\infty} \lambda(\Gamma(s)) & =\lambda^{\star} .
\end{aligned}
$$

Furthermore, equation (A10) implies

$$
\begin{aligned}
\lim _{s \rightarrow \infty} f(\lambda(\Gamma(s))) & =\lim _{s \rightarrow \infty}\left\{\frac{e^{2 s}}{2 m} g^{I J}\left(x_{0}, \phi^{\star}\right) v_{I} v_{J}+O\left(e^{s}\right)\right\} \\
& =\infty .
\end{aligned}
$$

But recall from (A13) that $f$ is strictly increasing over $\Lambda_{p_{\phi} \neq 0}$; hence (A18) requires

$$
\lim _{s \rightarrow \infty} \lambda(\Gamma(s))=\sup \left\{\Lambda_{p_{\phi} \neq 0}\right\} \in \mathbb{R} \cup\{\infty\} .
$$

Moreover, because $\Lambda$ is the closure of $\Lambda_{p_{\phi} \neq 0}\left(T^{*} \mathcal{U}_{\phi}\right.$ is the closure of $\left[T^{*} \mathcal{U}_{\phi}\right]_{p_{\phi} \neq 0}$, and $\lambda$ is continuous) we have $\sup \left\{\Lambda_{p_{\phi} \neq 0}\right\}=\sup \{\Lambda\}$ and hence

$$
\lim _{s \rightarrow \infty} \lambda(\Gamma(s))=\sup \{\Lambda\} .
$$

We can now assemble our results: recalling (A16) and noting that the continuous function $\lambda(\Gamma(\cdot))$ has limits (A17) and (A20), we deduce

$$
\Lambda_{p_{\phi} \neq 0} \supseteq \lambda(\Gamma(\mathbb{R})) \supseteq\left(\lambda^{\star}, \sup \{\Lambda\}\right) .
$$

(Note that the interval on the right is open: $\Lambda_{p_{\phi} \neq 0}$ does not contain $\lambda^{\star}$ or $\sup \{\Lambda\}$ in general.) Combining (A21) with (A13) we have

$$
\frac{\mathrm{d} f}{\mathrm{~d} \lambda}>0 \quad \forall \lambda \in\left(\lambda^{\star}, \sup \{\Lambda\}\right),
$$

but as this is true for all $\lambda^{\star} \in \Lambda$, we conclude that

$$
\frac{\mathrm{d} f}{\mathrm{~d} \lambda}>0 \quad \forall \lambda \in(\inf \{\Lambda\}, \sup \{\Lambda\}) .
$$

Hence $f$ is invertible.
For $d \geq 2$, we can therefore rewrite (A10) as

$$
\lambda\left(\phi, p_{\phi}\right)=f^{-1}\left(\frac{1}{2 m} g^{I J}\left(x_{0}, \phi\right) p_{\phi^{I}} p_{\phi^{J}}+V_{0}\left(x_{0}, \phi\right)\right),
$$

and substitute this into equation (A9):

$$
\begin{aligned}
& \frac{1}{2 m} g^{I J}(x, \phi) p_{\phi^{I}} p_{\phi^{J}}+V_{0}(x, \phi) \\
& =V_{\mathrm{cl}}\left(x, f^{-1}\left(\frac{1}{2 m} g^{I J}\left(x_{0}, \phi\right) p_{\phi^{I}} p_{\phi^{J}}+V_{0}\left(x_{0}, \phi\right)\right)\right) \\
& \equiv \tilde{V}_{\mathrm{cl}}\left(x, \frac{1}{2 m} g^{I J}\left(x_{0}, \phi\right) p_{\phi^{I}} p_{\phi^{J}}+V_{0}\left(x_{0}, \phi\right)\right) .
\end{aligned}
$$

But the only way this equation can hold for all $p_{\phi}$ is if the function on the right is a first-order polynomial in its second argument: $\tilde{V}_{\mathrm{cl}}(x, y)=\alpha(x)+\beta(x) \times y$ for some $\alpha, \beta:\left(x_{-}, x_{+}\right) \rightarrow \mathbb{R}$. Thus (A25) becomes

$$
\begin{aligned}
& \frac{1}{2 m} g^{I J}(x, \phi) p_{\phi^{I}} p_{\phi^{J}}+V_{0}(x, \phi) \\
& =\alpha(x)+\beta(x)\left(\frac{1}{2 m} g^{I J}\left(x_{0}, \phi\right) p_{\phi^{I}} p_{\phi^{J}}+V_{0}\left(x_{0}, \phi\right)\right),
\end{aligned}
$$

and comparing the coefficients of $p_{\phi^{I}} p_{\phi^{J}}$, we see that

$$
g^{I J}(x, \phi)=\beta(x) g^{I J}\left(x_{0}, \phi\right) .
$$

Of course, the metric on the left cannot depend on our choice of $x_{0}$, so neither can the combination on the right. ${ }^{4}$ Hence we can write (A27) as

$$
g^{I J}(x, \phi)=[b(x)]^{-2} \tilde{g}^{I J}(\phi),
$$

and conclude that the metric has the form (24) as claimed.

We can also compare the terms in (A26) that are independent of $p_{\phi}$ :

$$
\begin{aligned}
V_{0}(x, \phi) & =\alpha(x)+\beta(x) V_{0}\left(x_{0}, \phi\right) \\
& \equiv V_{x}(x)+[b(x)]^{-2} V_{\phi}(\phi),
\end{aligned}
$$

revealing that the potential can be slightly more general than the $V_{0}(x)$ considered in the main text. It is easy to introduce the new term $[b(x)]^{-2} V_{\phi}(\phi)$ into the analysis of section III: one simply adds $V_{\phi}$ to the definition of $E_{\phi}$ at the classical level (25) and the quantum level (31). This has no effect on the quantum correction (39).

All that remains is to deal with the special case $d=1$. As $\left[T^{*} \mathcal{U}_{\phi}\right]_{p_{\phi} \neq 0}$ is disconnected, $\Lambda_{p_{\phi}>0} \equiv \lambda\left(\left[T^{*} \mathcal{U}_{\phi}\right]_{p_{\phi}>0}\right)$

\footnotetext{
4 The function $\beta(x)$ implicitly depends on the choice of $x_{0}$ because $f(\cdot) \equiv V_{\mathrm{cl}}\left(x_{0}, \cdot\right)$ was used to construct $\tilde{V}_{\mathrm{cl}}$. This implicit dependence must cancel the explicit dependence of $g^{I J}\left(x_{0}, \phi\right)$ in (A27).
} 
may not connect to $\Lambda_{p_{\phi}<0} \equiv \lambda\left(\left[T^{*} \mathcal{U}_{\phi}\right]_{p_{\phi}<0}\right)$ and we might have

$$
\begin{aligned}
& \frac{\mathrm{d} f}{\mathrm{~d} \lambda}>0 \quad \forall \lambda \in \Lambda_{p_{\phi}>0}, \\
& \frac{\mathrm{d} f}{\mathrm{~d} \lambda}<0 \quad \forall \lambda \in \Lambda_{p_{\phi}<0},
\end{aligned}
$$

or vice versa. In this case, $f$ will not be invertible, but we can still define partial inverses $f_{+}^{-1}: f\left(\Lambda_{p_{\phi} \geq 0}\right) \rightarrow \Lambda_{p_{\phi} \geq 0}$ and $f_{-}^{-1}: f\left(\Lambda_{p_{\phi} \leq 0}\right) \rightarrow \Lambda_{p_{\phi} \leq 0}$ using the same arguments as $d \geq 2$. We would then write

$$
\begin{aligned}
\lambda\left(\phi, p_{\phi}\right) & =f_{\operatorname{sign}\left(p_{\phi}\right)}^{-1}\left(f\left(\lambda\left(\phi, p_{\phi}\right)\right)\right) \\
& =f_{\operatorname{sign}\left(p_{\phi}\right)}^{-1}\left(\frac{1}{2 m} g^{I J}\left(x_{0}, \phi\right) p_{\phi^{I}} p_{\phi^{J}}+V_{0}\left(x_{0}, \phi\right)\right),
\end{aligned}
$$

instead of (A24) and use this in (A25). Thus, $\tilde{V}_{\mathrm{cl}}$ becomes $\tilde{V}_{\mathrm{cl}}^{\operatorname{sign}\left(p_{\phi}\right)}$, but seeing as the left-hand side of equation (A25) is invariant under $p_{\phi} \rightarrow-p_{\phi}$, we must have $\tilde{V}_{\mathrm{cl}}^{+}=\tilde{V}_{\mathrm{cl}}^{-}$anyway. From then on, the argument proceeds exactly as above.
[1] B. S. DeWitt, Dynamical theory in curved spaces. I. A review of the classical and quantum action principles, Rev. Mod. Phys. 29 (1957) 377-397.

[2] C. DeWitt-Morette, K. D. Elworthy, B. L. Nelson, G. S. Sammelman, A stochastic scheme for constructing solutions of the Schrödinger equations, Annales de l'I.H.P. Physique thorique 32 (4) (1980) 327-341.

[3] M. Baszak, Z. Domaski, Canonical quantization of classical mechanics in curvilinear coordinates. Invariant quantization procedure, Annals of Physics 339 (2013) 89 108.

[4] H. Jensen, H. Koppe, Quantum mechanics with constraints, Annals of Physics 63 (2) (1971) 586 - 591.

[5] R. C. T. da Costa, Quantum mechanics of a constrained particle, Phys. Rev. A 23 (1981) 1982-1987.

[6] E. O. Silva, S. C. Ulhoa, F. M. Andrade, C. Filgueiras, R. Amorim, Quantum motion of a point particle in the presence of the Aharonov-Bohm potential in curved space, Annals of Physics 362 (2015) 739 - 751.

[7] C. Ortix, Quantum mechanics of a spin-orbit coupled electron constrained to a space curve, Phys. Rev. B 91 (2015) 245412.
[8] Y.-L. Wang, H. Jiang, H.-S. Zong, Geometric influences of a particle confined to a curved surface embedded in three-dimensional Euclidean space, Phys. Rev. A 96 (2017) 022116.

[9] C. G. Callan, S. Coleman, R. Jackiw, A new improved energy-momentum tensor, Annals of Physics 59 (1) (1970) $42-73$.

[10] A. Karamatskou, H. Kleinert, Geometrization of the Schrödinger equation: Application of the Maupertuis principle to quantum mechanics, International Journal of Geometric Methods in Modern Physics 11 (08) (2014) 1450066 .

[11] C. Kiefer, Quantum Gravity, 3rd Edition, Oxford University Press, 2012.

[12] R. P. Feynman, Space-time approach to non-relativistic quantum mechanics, Rev. Mod. Phys. 20 (1948) 367-387.

[13] H. Kleinert, Path Integrals in Quantum Mechanics, Statistics, Polymer Physics, and Financial Markets, 5th Edition, World Scientific, 2009.

[14] S. Storchak, Path integrals on warped product manifolds, Physics Letters A 174 (1) (1993) 13 - 18. 